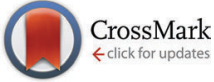

Cite this: New J. Chem., 2017 41,1723

Received 29th November 2016 Accepted 4th January 2017

DOI: 10.1039/c6nj03716

rsc.li/njc

\section{Microwave treated sol-gel synthesis and characterization of hybrid ZnS-RGO composites for efficient photodegradation of dyes}

\author{
Lellala Kashinath, ${ }^{a}$ Keerthiraj Namratha, ${ }^{a}$ Shivanna Srikantaswamy, ${ }^{a}$ Ajayan Vinu ${ }^{b}$ \\ and Kullaiah Byrappa*a
}

\begin{abstract}
In the present work, assembly of nanosized ZnS particles grown on the surface of a 2D platform of reduced graphene oxide (RGO) sheets by the sol-gel method without the use of any surfactant/stabilizing agents and treatment with a microwave irradiation technique was reported. During this process, the RGO sheets act as the substrate, matrix and fine template for the growth/decoration of isometric ZnS nanoparticles and graphene sheets were formed simultaneously. The synthesized nanocomposites were used as photocatalyst materials for the degradation of Brilliant Blue and Brilliant Yellow dyes under UV light radiation. The results signify that the nanocomposites exhibit an enhanced photocatalytic activity compared to PZnS and RGO, owing to the high specific surface area, and the reduction of photoinduced electron-hole pair recombination in ZnS, which are due to the inclusion of RGO and its synergetic effects. Reduced graphene oxide acts as an excellent electron transporting material and effectively suppresses the charge recombination of ZnS-RGO nanocomposites exhibiting much higher photoresponse activity. The chemical composition, morphology and microstructural features of the materials were characterized by $\mathrm{X}$-ray diffraction, FTIR, UV-Vis spectroscopy, photoluminescence, Raman and energy dispersive spectroscopy, FE-SEM, XPS, and TEM.
\end{abstract}

\section{Introduction}

Heterogeneous photocatalysis offers great potential for environmental purification by converting photon energy into chemical energy. ${ }^{1-3}$ The recombination of photogenerated charge carriers has faster kinetics than surface redox reactions and greatly reduces the quantum efficiency of photocatalysis. ${ }^{4-6}$ To enhance the photocatalysis efficiency, it is vital to retard the recombination of the charge carriers. Semiconductor nanoparticles are an important topic of interest because of their unique optical and electronic properties owing to the quantum confinement effect and large surface area. ${ }^{7,8}$ These nanoparticles have an increased band gap compared to their bulk counterparts leading to a blue/red shift in the absorption/emission spectra. The bandgaps of the nanoparticles can be tuned by controlling the size and morphology, and thus can be modified for various potential applications. ${ }^{9,10}$ Among semiconductors, zinc sulphide

\footnotetext{
${ }^{a}$ Centre for Materials Science and Technology, University of Mysore, Vijnana Bhavan, P.B. No. 21, Manasagangothri, Mysore -570006, India. E-mail:kbyrappa@gmail.com

${ }^{b}$ Future Industries Institute, Division of Information Technology, Engineering and Environment, University of South Australia, Building X-X2-09,

Mawson Lakes Campus, Mawson Lakes 5095, Australia
}

is a non-toxic II-VI semiconductor material with a wide direct band gap energy of $3.71 \mathrm{eV}$ and $3.78 \mathrm{eV}$ for a cubic zinc blend and a hexagonal wurtzite structure, respectively, with an excitonic binding energy of $40 \mathrm{meV}$ at room temperature. ${ }^{11,12}$

ZnS has great potential applications in sensors, catalysis and optical properties. It also haswide emerging applications in optoelectronic devices, light emitting diodes, fuel cells, solar energy conversions, photocatalysis, efficient phosphor inflated panel displays, cathode ray tubes, luminescent materials, piezoelectric materials and thin films of electroluminescent devices $^{13,14}$ and has been extensively studied because of its size, shape, and morphology dependent quantum confinement effects. ${ }^{15}$

Generally, in order to avoid ZnS nanoparticle agglomeration, synthetic polymers, microgels, mesoporous silicate materials and other organic/surfactant stabilizers are adopted as stabilizing or supporting materials to keep the size of $\mathrm{ZnS}$ in the nano-range. However, in most cases, the pure products are obtained only after the complete removal of the surfactants, which makes the whole process more complicated. Therefore, it is difficult to control the growth and agglomeration of particles during synthesis without the use of any surfactant/stabilizer, and template-free self-assembled $\mathrm{ZnS}$ architectures with high photocatalytic efficiency are still difficult to achieve and rarely reported. On the other hand, the quick recombination of photogenerated charge 
carriers in $\mathrm{ZnS}$ nanocrystals limits their photocatalytic properties and decreases the efficiency rate in optoelectronics fields. Additionally, loading semiconductor particles on the surface of graphene is a good way to prevent the aggregation of the semiconductor nanoparticles and the stacking of graphene sheets.

Graphene is an emerging material of monolayered $\mathrm{sp}^{2}$ hybrid carbon atoms in a two-dimensional hexagonal honeycomb structure and when conjugated with chemicals can cause a broad spectrum of attractive properties, such as optical transparency, high electroconductivity, ${ }^{16}$ mechanical flexibility and strong thermal/chemical stability. ${ }^{17}$ Recently, graphenebased materials decorated with metal or metal oxide/metal sulphide nanoparticles have attracted a great deal of attention due to their potential applications in many science and technological fields. ${ }^{18}$ Nanocomposites composed of graphene and various semiconductors or metals/metal oxides show significantly improved properties compared to pure semiconductor nanomaterials in electrocatalysis, super capacitors, lithium ion batteries and photocatalytic reactions. ${ }^{19}$ In addition, forming nanocomposites can effectively improve the photoelectric characteristics of the semiconductor and functionalized graphene due to the emission tunability and photoconductive characteristics of the semiconductor nanoparticles. Therefore, nanocomposites provide semiconductor nanoparticles with excellent emission tunability and graphene with superior electron mobility. ${ }^{20,21}$

In the present work, ZnS-RGO nanocomposites synthesized by a simple sol-gel method without the use of any surfactant or stabilizing agents, with zinc sulphate and sodium sulphide as sulphur sources, and treatment with microwave irradiation are reported. Here, ZnS acts as an excellent photocatalyst and its photo-responsive nature helps to excite electrons from the valence band to the conduction band and to create electronhole pairs which can migrate and initiate redox reactions with water and oxygen. It then degrades organic molecules or reduces metal ions absorbed on the surface of $\mathrm{ZnS}$ owing to their large shape anisotropy and superior thermal/chemical stability in ambient environments. ${ }^{22}$ The composites of graphene and $\mathrm{ZnS}$ nanoparticles prevent graphene from aggregating due to the distribution of $\mathrm{ZnS}$ nanoparticles on the surface of graphene sheets, improving the photoelectric characteristics of the $\mathrm{ZnS}$ semiconductor nanoparticles due to the electron capture and transferability of graphene resulting in a more efficient separation of the photo-excited charge carriers from the ZnS-RGO composites. $^{23}$ Microwave irradiation-assisted chemical transformations are pollution free, eco-friendly and offer high yields together with simplicity in processing and handling.

Microwave energy, in contrast, is introduced into the chemical reactor remotely and passes through the walls of the reaction vessel, heating the reactants and solvents directly and using microwave energy instead of conventional heating often results in good yields in a short time as compared with reactions by classical synthetic methods. Microwave radiation provides a fast and uniform heating rate that causes rapid particle nucleation and growth which can reduce the reaction time, and helps in facilitating porosity, therefore, saving a large amount of energy. ${ }^{24,39,68}$
The effect of microwave irradiation in chemical reactions is a combination of the thermal effect and non-thermal effects, i.e., overheating, hot spots and selective heating, and non-thermal effects of the highly polarizing field, in addition to effects on the mobility and diffusion that may increase the probabilities of effective contacts. Microwave (MW) reactions involve selective absorption of MW energy by polar molecules, non-polar molecules being inert to MW dielectric loss. Microwave dielectric heating drives chemical reactions by taking advantage of the ability of some liquids and solids to transform electromagnetic radiation into heat wherein chemical reactions are accelerated because of selective absorption of microwave energy by the polar molecules. The penetration properties of microwave electromagnetic irradiation through solutions should result in uniform heating of the reaction solution, giving uniform nucleation and growth rates, as compared with thermal convection. ${ }^{25,26,69}$

\section{Results and discussion}

\subsection{Crystalline structure}

The crystalline structures and phase orientations of RGO nanosheets, PZnS nanoparticles, and hybrid ZnS-RGO nanocomposites have been investigated by XRD analysis as shown in Fig. 1. The diffraction patterns of RGO show an intense and sharp peak at $2 \theta=23.9^{\circ}$ attributed to the (100) lattice plane with a corresponding " $d$ " spacing of $0.83 \mathrm{~nm}$. A peak at $16^{\circ}$ corresponding to the (001) plane and a small minor hump located at $43^{\circ}$ for the (101) planes as shown in Fig. 1a indicate the presence of few-layered graphene sheets, which means that RGO sheets have been effectively exfoliated from the raw graphite. Further, the reduction of reduced graphene oxide to make nanocrystalline graphene in the zinc sulphide composite (ZnS-RGO) is observed by studying the crystalline phase behaviour as shown in Fig. 1. The XRD spectrum of the ZnS-RGO composites shows the peaks of isometric/cubic ZnS nanoparticles superimposed on the surface of $2 \mathrm{D}$ lattice phases of reduced graphene oxide/graphene sheets. The peaks at $28.5^{\circ}, 33.5^{\circ}, 48.2^{\circ}, 56.8^{\circ}$, and $76.8^{\circ}$ are the corresponding lattice planes of references at (111), (200), (220), (311) and (422) as shown in Fig. 1(c-e) for GZ1, GZ2 and GZ3, respectively, which matches well with the cubic structure

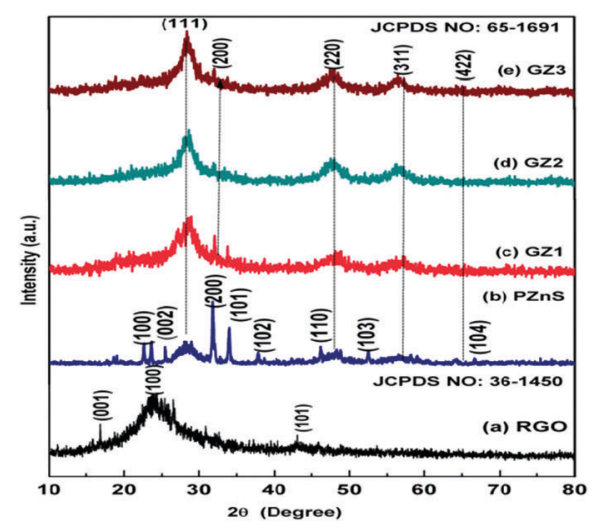

Fig. 1 XRD patterns of (a) RGO, (b) PZnS, (c) GZ1, (d) GZ2 and (e) GZ3. 
Table 1 The 20, (hkl), FWHM, D spacing, crystalline size, dislocation density and strain for the major indexing peak of as-prepared RGO, PZnS, and ZnSRGO composites

\begin{tabular}{lllllll}
\hline Sample & $2 \theta,(h k l)$ & $\begin{array}{l}\text { FWHM } \\
\text { (radian) }\end{array}$ & $D$ spacing & $\begin{array}{l}\text { Crystalline } \\
\text { size }(\mathrm{nm})\end{array}$ & $\begin{array}{l}\text { Dislocation density } \\
(\delta) \times\left(10^{16}\right) \operatorname{lines~}^{2} \mathrm{per}^{2}\end{array}$ \\
\hline RGO & $23.9,(002)$ & 7.134 & 3.17 & 1.50 & 7.711 & $\begin{array}{l}S \text { strain } \\
(\varepsilon) \times 10^{-3}\end{array}$ \\
PZnS & $28.5,(111)$ & 2.138 & 3.12 & 4.03 & 6.979 & 0.93 \\
GZ1 & $27.9(111)$ & 4.367 & 3.19 & 1.96 & 2.843 & 7.444 \\
GZ2 & $28.6,(111)$ & 2.238 & 3.11 & 3.86 & 8.572 & 0.94 \\
GZ3 & $28.7,(111)$ & 2.402 & 3.10 & 3.56 & & 1.01
\end{tabular}

of face-centred crystal phase (FCC) (JCPDS No 65-1691) with the lattice constant parameters of $a=b=c=5.410 \AA$. The peaks in the diffractogram of PZnS at $2 \theta$ values of $23.9^{\circ}, 26.9^{\circ}, 28.5^{\circ}, 33.1^{\circ}$, $35.7^{\circ}, 39.5^{\circ}, 47.5^{\circ}, 51.7^{\circ}, 56.4^{\circ}, 59.2^{\circ}, 69.5^{\circ}$, and $76.8^{\circ}$ can be indexed to (100), (002), (111), (200), (101), (102), (110), (220), (103), (311), (422), and (104), respectively, as shown in Fig. 1b, which are the lattice planes of hexagonal $\mathrm{ZnS}$ with primitive cubic cell phase (JCPDS Card No 36-1450) with lattice constants of $a=b=3.820 \AA$ and $c=6.527 \AA$. The nanocomposite crystalline size, FWHM, $d$ spacing with $(h k l)$ for the major intensity peak at (111) along with dislocation density and strains are given in Table 1.

The crystallite size $(D)$, dislocation density $(d)$ and strain $(\varepsilon)$ are calculated by using the following relations,

$$
D_{h k l}=\frac{K \lambda}{\beta \cos \theta}, \delta=\frac{1}{D 2} \text { and } \varepsilon=\frac{\beta \cos \theta}{4}
$$

where $\lambda=1.5444 \AA$ A represents the wavelength of the X-rays, $\theta$ is the diffraction angle, and $\beta$ is the full width at half maximum (FWHM) of the diffraction peak.

\subsection{FTIR analysis}

To assess the reduction of RGO and the formation of the composites, Fig. 2 shows the FTIR spectral analysis for the identification of molecular absorption on the surface and to ascertain the functional groups of the RGO nano sheets, PZnS nanoparticles, and ZnS-RGO nanocomposites. The functional group of RGO showing a strong and broad absorption peak value at $3410 \mathrm{~cm}^{-1}$ is due to hydroxyl $(\mathrm{O}-\mathrm{H}) ; 1625 \mathrm{~cm}^{-1}(\mathrm{C}=\mathrm{C})$

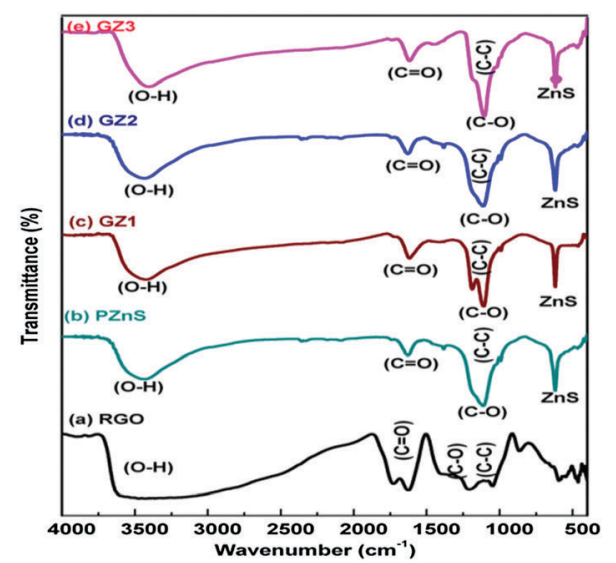

Fig. 2 FTIR spectra of (a) RGO, (b) PZnS, (c) GZ1, (d) GZ2 and (e) GZ3. and $1590 \mathrm{~cm}^{-1}(\mathrm{C}=\mathrm{O})$ are the aromatic stretching vibration of $\mathrm{sp}^{2}$ hybridized molecules. The peak at $1731 \mathrm{~cm}^{-1}$ is due to carbonyl stretching vibration, the $\mathrm{O}-\mathrm{H}$ bending vibration of $(\mathrm{C}=\mathrm{OH})$ is at $1350 \mathrm{~cm}^{-1}$, the peak at $1205 \mathrm{~cm}^{-1}$ is due to $(\mathrm{C}=\mathrm{OH})$ stretching and the epoxy $(\mathrm{C}-\mathrm{O})$ stretching vibration is at $1051 \mathrm{~cm}^{-1}$ as shown in Fig. $2 \mathrm{a}^{28}$ The FTIR spectra of the nanocomposite show the presence of a skeletal vibration absorption peak between $1570-1600 \mathrm{~cm}^{-1}$ corresponding to graphene suggesting that RGO is successfully reduced to graphene (Nethravathi et al. $2009,{ }^{29}$ Lv et al. $2012^{30}$ ). The characteristic absorbance peaks of the PZnS and ZnS-RGO nanocomposites located at $3410 \mathrm{~cm}^{-1}$ are due to hydroxyl groups $(\mathrm{O}-\mathrm{H})$; the presence of peaks at $1595-1620 \mathrm{~cm}^{-1}$ for carboxylic $(\mathrm{C}=\mathrm{O})$ indicates the reduction of reduced graphene oxide to graphene. Peaks at $1045 \mathrm{~cm}^{-1}$ due to (C-O) epoxy stretching and $1205 \mathrm{~cm}^{-1}(\mathrm{C}=\mathrm{OH}$ stretching $)$ and $615 \mathrm{~cm}^{-1}$ due to the presence of $\mathrm{ZnS}$ (metallic sulphide bonds) are clearly shown in Fig. 2(b-e) for PZnS, GZ1, GZ2, and GZ3. ${ }^{31}$

\subsection{Raman vibrations}

Raman spectroscopy is a useful tool to investigate the significant carbon purity level, hybridization and structural changes of RGO to the ZnS-RGO composite. The D band is attributed to the disorder of graphene, resulting from the breathing mode of symmetry which is caused by phonon interaction near the K-edge zone boundary, whereas the $\mathrm{G}$ band is attributed to the ordered $\mathrm{E}_{2 \mathrm{~g}}$ phonon mode vibrations at the Brillouin Zone centre of $\mathrm{sp}^{2}$ bonded carbon atoms. The intensity ratio of $\mathrm{D}$ to $\mathrm{G}\left(I_{\mathrm{D}} / I_{\mathrm{G}}\right)$ related to the density of defects in the RGO to composites are used to measure the quality of graphitization or defective disorders in crystalline graphene. The Raman peaks of RGO can be found at $1351 \mathrm{~cm}^{-1}$ and $1595 \mathrm{~cm}^{-1}$ corresponding to D and G bands respectively. ${ }^{32}$ Raman lines show substantial broadening, phonon softening and line broadening of the Raman peaks which can be attributed to quantum confinement effects. ${ }^{33}$ Furthermore, the peaks at $645 \mathrm{~cm}^{-1}$ ascribed to pure $\mathrm{ZnS}$ are due to longitudinal optical (LO) phonons and the weaker peak at $445 \mathrm{~cm}^{-1}$ is associated with the transverse optical (TO) phonons. The peaks of first-order LO and TO phonons from the ZnS nanostructure exhibit a shift towards the lower energy. When the material structure reduces to nanosize or the lattice distortion increases, the wave-vector selection rule for the first order Raman scattering is relaxed and the photon scattering will not be limited to the centre of the Brillouin zone, so the phonon dispersion near the centre of the Brillouin zone is considered for confirmation of hybridization. ${ }^{34}$ 


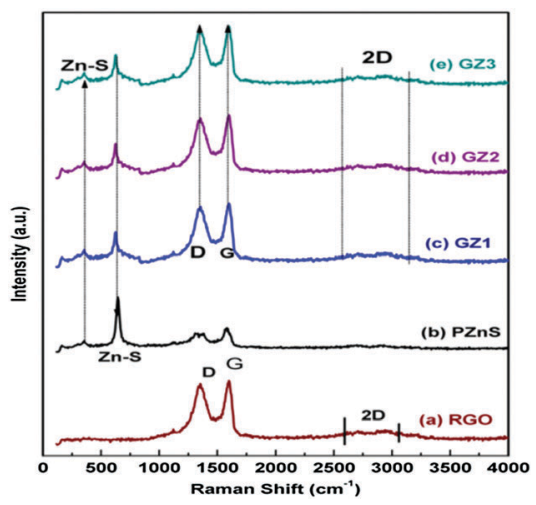

Fig. 3 Raman spectra of (a) PZnS, (b) GZ1, (c) GZ3 and (d) RGO.

The results confirm the presence of both $\mathrm{ZnS}$ and RGO in the ZnS-RGO composites as shown in Fig. 3(c-e) for the GZ1, GZ2, and GZ3 samples. In addition, the variation of $I_{\mathrm{D}} / I_{\mathrm{G}}$ with respect to the oxidation level shows an increase of the $I_{\mathrm{D}} / I_{\mathrm{G}}$ ratio indicating lower oxidation levels (that is to say higher reduction levels). ${ }^{33}$ However, the major evidence is the degree of disorder in RGO, which can be observed from the intensity ratio of the $\mathrm{D}$ and $\mathrm{G}$ bands $\left(I_{\mathrm{D}} / I_{\mathrm{G}}\right)$. From Fig. 3 it can be inferred that the ratio of the ZnS-RGO composite is higher compared to RGO, implying that the microwave irradiation reaction has created a large number of $\mathrm{sp}^{2}$ bonds and less structural defects in the RGO lattice. Furthermore, we see that the ZnS-RGO composite displays surface enhanced Raman scattering activity (SERS) in comparison with those of the original RGO under the same test conditions, and no signals of $\mathrm{ZnS}$ are detected in the ZnS-RGO composites. In most semiconductors, the second mechanism is predominant for SERS. The enhancement involves the chemisorption interaction and the semiconductor/absorbance charge transfer enhancement should be the predominant mechanism responsible for the observed enhancement of Raman intensity. ${ }^{35,36}$

\subsection{Optical studies}

2.4.1. UV-Vis spectroscopy analysis. The optical diffuse reflectance absorption spectra of RGO, PZnS, and the hybrid ZnS-RGO composites are shown in Fig. 4A. The absorption
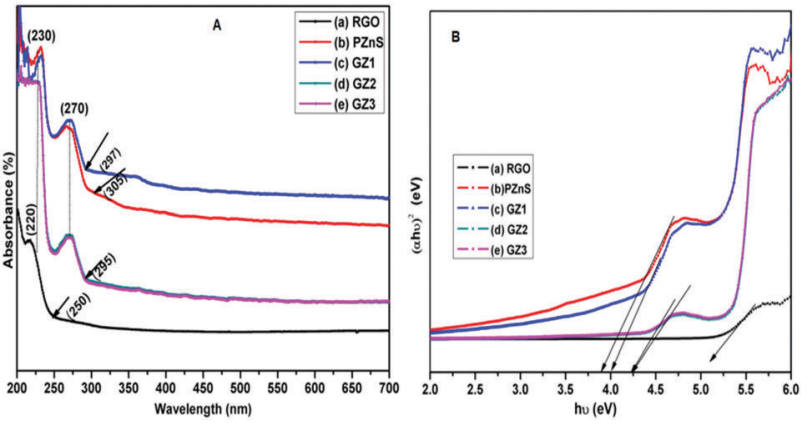

Fig. 4 (A) UV-Vis spectra of (a) RGO, (b) PZnS, (c) GZ1, (d) GZ2 and (e) GZ3. (B). Tauc's plot of (a) RGO, (b) PZnS, (c) GZ1, (d) GZ2 and (e) GZ3. spectrum of RGO exhibits a maximum peak value at $220 \mathrm{~nm}$ $(5.6 \mathrm{eV})$ and $250 \mathrm{~nm}(4.96 \mathrm{eV})$ corresponding to the $\mathrm{n}-\pi^{*}$ transition of $\mathrm{C}-\mathrm{C}$ and $\mathrm{C}=\mathrm{C}$ bonds which indicates the narrowing/ broadening of the band gap as shown in Fig. 4A(a). The maximum absorption spectra of the nanocomposites were located at $230 \mathrm{~nm}$ $(5.3 \mathrm{eV})$ and $270 \mathrm{~nm}(4.5 \mathrm{eV})$ corresponding to $\mathrm{n}-\pi^{*}$ transitions as shown in Fig. 4A(b-e) for PZnS, GZ1, GZ2 and GZ3. The optical percentage of the transmissions is between $85-94 \%$ for the nanocomposites. The shoulder peak of the spectra corresponding to the fundamental absorption edges in the samples was used to estimate the band gap of the materials. ${ }^{37,38}$

$$
E_{\mathrm{g}}=h \nu=h c / \lambda
$$

where $h=$ Planck's constant, $c=$ velocity of light, $\lambda=$ wavelength of absorption and $E=$ energy of the band gap of the semiconducting nanoparticles in the optical spectra. When the ZnS crystal reacts with a photon, which carries energy equal to $h$ or more than the bandgap energy of the $\mathrm{ZnS}$ nanocrystals, it makes an electron excited from the valence band to the conduction band, consequently resulting in a positive hole in the valence band and recombination of electron and holes that plays a vital role in optoelectronics fields. Due to confinement of both electrons and holes, the lowest energy optical transition from the valence to conduction band will increase, effectively increasing the band gap $\left(E_{\mathrm{g}}\right)$. The calculated bandgap values of the nanocomposites with respect to the absorbance spectra are $275 \mathrm{~nm}(4.15 \mathrm{eV}), 310 \mathrm{~nm}(4.0 \mathrm{eV})$ and $315 \mathrm{~nm}(3.9 \mathrm{eV})$, respectively, for GZ3, GZ2, GZ1 and PZnS which is blue shifted from that of bulk $\mathrm{ZnS} 340 \mathrm{~nm}(3.65 \mathrm{eV})$ and the band gap for the RGO is $5 \mathrm{eV}$ as shown in Fig. $4 \mathrm{~B}$.

Increasing of the band gap energies of composite nanostructures could be an indication of the quantum confinement effect due to the decrease in the size and structures in $\mathrm{ZnS}$ and due to the interfacial action of surface plasmons of reduced graphene oxide and PZnS and increased band gap compared to bulk ZnS. ${ }^{39}$ The band gap energy of the nanomaterials is obtained from the absorption maxima and according to quantum confinement theory, electrons in the conduction band and holes in the valence band are spatially confined by the potential barrier of the surface. The band gap broadening of $\mathrm{ZnS}$ can be attributed to the two integrative factors and the presence of sulphur vacancies can create adulteration levels near the valence band (VB), thereby inducing band gap narrowing/broadening, and the close interfacial interaction between ZnS and RGO could contribute to the band gap narrowing.

There is a slight decrease in the bandgap value for higher concentrations of ZnS in ZnS-RGO composites due to a higher amount of sulphur, which shields the activator $\mathrm{Zn}$; sulphur acts as an accelerated vulcanizer for cross linking of $\mathrm{Zn}$ which is proactive in the UV-Vis region leading to slight alteration in the band gap but effectively active in the UV region and makes it active in the visible light region. ${ }^{40}$ This result indicates that band gap narrowing of $\mathrm{ZnS}$ are is due to coupling between RGO and $\mathrm{ZnS}$, and under UV light irradiation, semiconductor ZnS in the RGO-ZnS nanocomposites can be bandgap photo-excited, thereby generating electron-hole pairs. The energy band gaps 
of the ZnS nanoparticles and nanocomposites were calculated using Tauc's relation in which a graph $(\alpha h \nu)^{2} v s$. $h \nu$ is plotted, where $\alpha$ is the absorption coefficient and $h \nu$ is the photon energy. The energy band gap $E_{\mathrm{g}}$ as determined by extrapolation of the linear portion of $(\alpha h \nu)^{2} v s$. $h \nu$ curves to $(\alpha h \nu)^{2}=0$ is shown in Fig. 4B.

2.4.2. Photoluminescence spectroscopy. Photoluminescence studies provide information on defect states and the electronic states in the bandgap of $\mathrm{ZnS}$ and RGO, which take part in irradiative de-excitation of the sample. In nanocrystals, the defect states may shift or the density could be increased as revealed by PL studies. The photoluminescence emission spectra of the PZnS and ZnS-RGO (directly producing ZnS nanoparticles on reduced graphene oxide) samples with an excitation wavelength of $350 \mathrm{~nm}$ at room temperature are shown in Fig. 5. The maximum emission spectra of the ZnS-RGO nanocomposite for the GZ1, GZ2 and GZ3 samples are at $452 \mathrm{~nm}(2.74 \mathrm{eV})$ attributed to sulphur vacancies and interstitial sulphur lattice defects or zinc in $\mathrm{ZnS}$, where the electrons recombine with holes in the valence band. ${ }^{41,42}$ Furthermore, the maximum PL emission spectrum of the $\mathrm{ZnS}-\mathrm{RGO}$ sample is blue-shifted to $485 \mathrm{~nm}(2.55 \mathrm{eV})$ which could be assigned to the size decrease of $\mathrm{ZnS}$ particles in the $\mathrm{ZnS}-\mathrm{RGO}$ nanocomposites as shown in Fig. 5(b-e). Broadening of the emission peak can be attributed to size distribution and the increase of the surface states owing to increase in the surface to volume ratio for smaller nanoparticles. The origin of the emission band centred at $490 \mathrm{~nm}$ is attributed to the Zn-vacancy. This phenomenon demonstrates that the emission energy of the surface states is corrected to the quantum-size effects. ZnS-RGO has a higher PL intensity, which is attributed to energy transfer from graphene to $\mathrm{ZnS}$. Electrons are excited by incident light from the valence band of ZnS to the conduction band. ${ }^{43}$ Because of the interaction between $\mathrm{ZnS}$ and graphene, the excited electrons can transfer to graphene sheets and get energy from the excitation levels of graphene, then the electrons obtained energy transfer from the excited state of graphene to $\mathrm{ZnS}$ and recombine with holes, leading to enhancement in the PL intensity. ${ }^{44}$

Further investigation of the luminescence mechanism causing the blue-green emission spectra monitored at a wavelength of $582 \mathrm{~nm}(2.13 \mathrm{eV})$ has been recorded and shown in Fig. 5. The bluegreen emission band can be excited both at energies near the

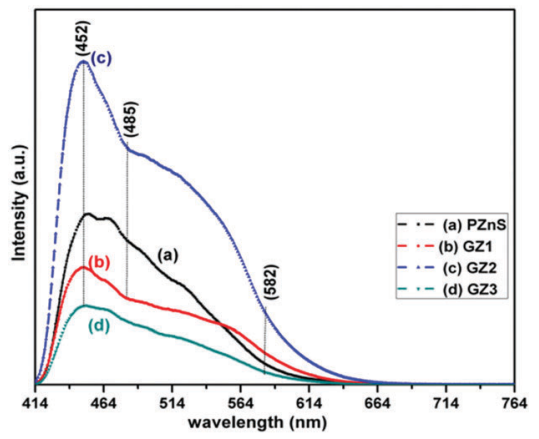

Fig. 5 PL spectra of (a) PZnS, (b) GZ1, (c) GZ2 and (d) GZ3. band edge of the $\mathrm{ZnS}$ (the UV region) and at energies below the band edge (the visible region).

\subsection{Morphology and chemical composition}

The morphology of pure RGO, PZnS and hybrid ZnS-RGO nanocomposites prepared by varying the sulphur sources characterized by FESEM, chemical composition and elemental analysis of the composites was investigated by EDS analysis, respectively, as shown in Fig. 6. The FESEM images of the RGO are curled and wrinkled corrugated nanosheets as shown in Fig. 6(A), while the PZnS shows the microspherical/cubical shape of uniform surface structures as shown in the inset of Fig. 6(E). Isometric/cubic ZnS nanoparticles are decorated uniformly on the surface of reduced graphene nanosheets and the two-dimensional structure of graphene sheets with micrometer long wrinkles can be clearly distinguished as shown in Fig. 6(B-D). The RGO nanosheets function as conductive bands for the interconnection between various $\mathrm{ZnS}$ microspheres/isometric structures and the transfer of photogenerated charge carriers for enhancement of the photocatalytic efficiency. The graphene sheets play an important role in assisting as template/matrix for $\mathrm{ZnS}$ nanoparticle growth, its dispersion on its surface and at the same time ZnS decoration helps to prevent the aggregation of the graphene sheets. ${ }^{45,46}$

Further, to investigate the microscopic structure and morphology information of the PZnS, RGO sheets and hybrid nanocomposites, transmission electron microscopy (TEM) analysis was performed to examine the surface interaction between RGO and the ZnS-RGO nanocomposites as shown in Fig. 7. The TEM image provides information on the limitation of the surface analysis and the large size of the microsphere. Fig. $7(\mathrm{a}-\mathrm{c})$ shows that there is a nice uniform dispersion of nanosized ZnS particles on the surface of the reduced graphene oxide sheet, which supports information about the limitation of the surface analysis. ${ }^{47}$ A more careful and close view reveals the presence of tiny ZnS nanoparticles, which are highly dispersed on the graphene surface. The images of RGO reveals the confirmation of nanosheets and the existence of layered sheet (si-, bi- and few layers) patterns observed as shown in Fig. 7d. Folded thin layers of graphene resembling crumpled silk veil waves with the approximate size of a few hundred square nanometres are clearly visible in the image. These sheets are transparent and very stable under the electron beam. The RGO nanosheets can function as conductive bands for the interconnection between various ZnS nanoparticles and the transfer of photo-generated charge carriers for enhancement of the photocurrent effect and the influence of graphene on the morphology. The light-gray nanoparticles are the RGO sheets, and the black dark regions on the RGO background are due to the presence of $\mathrm{ZnS}$ particles. The images of the $\mathrm{ZnS}$ nanoparticles in a cubic/ isometric structure are confirmed in all the composites (ZnS-RGO composite) GZ1, GZ2 and GZ3 as shown in Fig. 7(a-c). This indicates that the graphene sheets may play an important role in assisting ZnS nanoparticle growth and dispersion on its surface, and at the same time, ZnS-decoration helps to prevent the aggregation of the graphene sheets. ${ }^{48}$ 


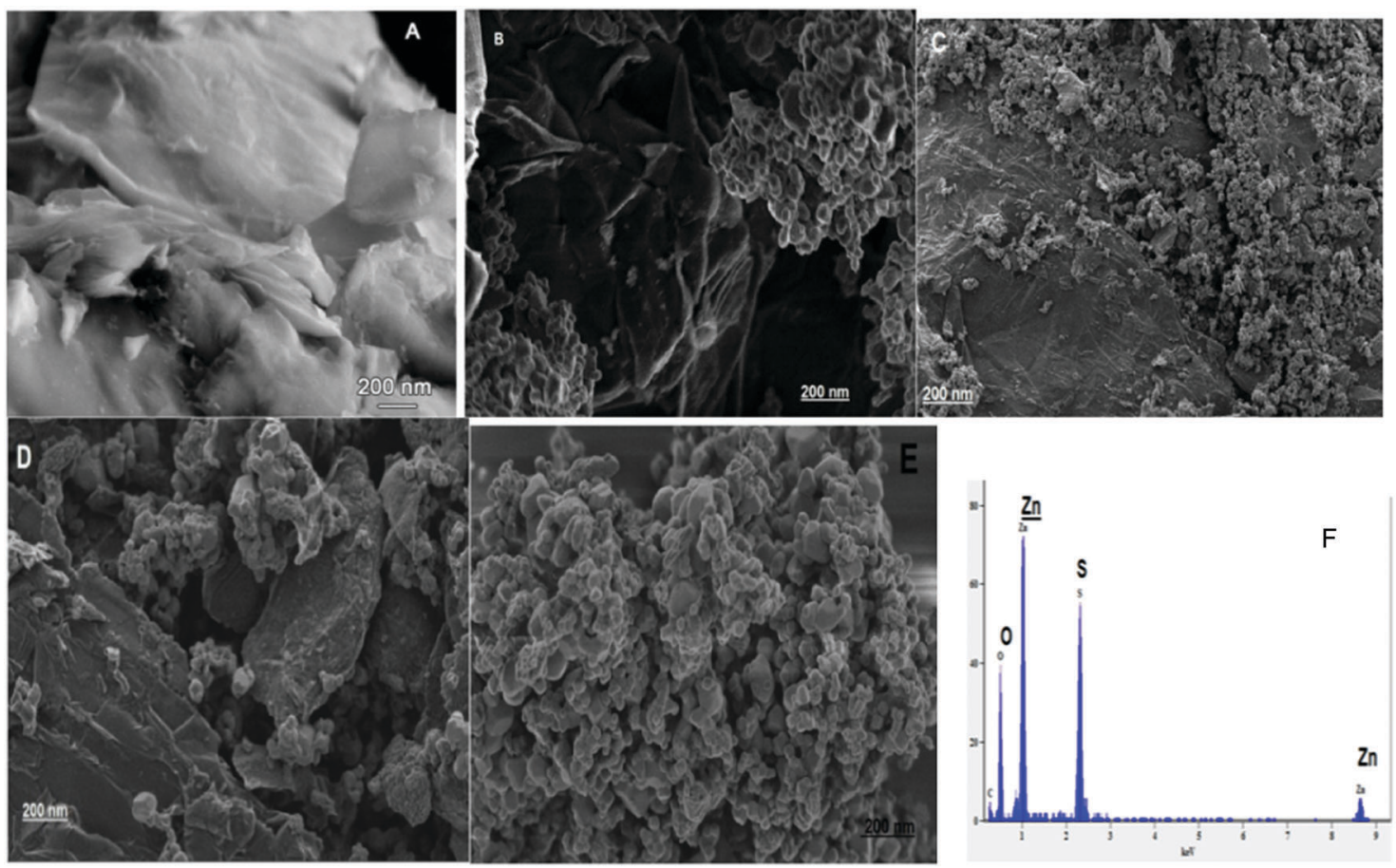

Fig. 6 FESEM image of (A) RGO, (B) GZ1, (C) GZ2, (D) GZ3 and (E) PZnS, and (F) EDS spectra analysis of the ZnS-RGO composite (GZ1).

The TEM image of RGO clearly shows the lattice fringes of ultra-thin crumpled sheets with size ranging between tens to several hundreds of square nanometers visible in the image suggesting that the well-defined crystal structure with lattice indices at (100) and (101) were observed in SAED as shown in the inset image of Fig. 7d. The SAED pattern of RGO shows the two-fold concentric ring pattern, RGO formed is crystalline as it shows sharp diffraction patterns confirming a more regular carbon framework. These sheets are transparent and very stable under the electron beam. On the other hand, the images of ZnS-RGO show that ZnS particles are embedded in reduced graphene oxide sheets. The SAED pattern of ZnS-RGO shows the six-fold pattern of ZnS arising due to graphene in addition to the ring pattern from $\mathrm{ZnS}$ particles, indicating the formation of a ZnS-RGO complex. The closed ring structure in the SAED pattern confirms the cubic crystalline phase of the sample and the results are in agreement with the XRD analysis. SAED image patterns as shown in the inset image of Fig. 7 shows a set of rings due to the random orientation of crystallites, which relate to diffraction from different planes of the $\mathrm{ZnS}$ nano crystallites. The SAED patterns show many rings, among them the strongest four rings correspond to the (111), (200), (220) and (311) planes of the cubic phase of $\mathrm{ZnS}$ as shown in the inset image of Fig. 7(a-c) for GZ1, GZ2 and GZ3, respectively, which is consistent with the values reported in the JCPDS (No 65-1691), and matches well with the diffraction results of XRD as shown in Fig. 1.

The nanoparticles' average particle size distribution of the ZnS-RGO composite analysis is shown in Fig. 7e. It can be seen from Fig. 7e that the size distribution of the particles shows a dual modal distribution of $35.8 \mathrm{~nm}$ (79\% volume, 24.1 width) for $\mathrm{ZnS}$ and $275 \mathrm{~nm}$ (21\% volume, 350 width) for RGO sheets confirming the presence of ZnS decorated on RGO sheets for GZ1 and $45.5 \mathrm{~nm}$ (85\% volume, 25 width) for $\mathrm{ZnS}$ and $200 \mathrm{~nm}$ (15\% volume, 330 width) for RGO, respectively, for the GZ3 composite. The particle size distribution of $\mathrm{ZnS}$ and RGO sheets reported in the present work is very low compared to the reported value of M. Sookhakian et al. (2013). ${ }^{55}$

The energy dispersive spectrometry (EDS) of RGO, PZnS, and the hybrid nanocomposite is analysed for the confirmation of chemical composition and atomic weight $\%$, where the atomic $\mathrm{Zn}: \mathrm{S}$ ratio is about $2: 1$ for GZ2 and GZ3, and $1: 1$ for GZ1 and is consistent with the stoichiometric ratio of the ZnS nanoparticles as can be seen in Table 2 of the EDS spectra. The atomic weight percentages of $\mathrm{Zn}$ and the sulphur ratio are shown in Table 2. Based on the results, the growth mechanisms of the $\mathrm{ZnS}-\mathrm{RGO}$ composites can be predicted. The $\mathrm{Zn}^{2+}$ positive ions gather on the negatively charged RGO surface. The microwave radiation process results in reaction of adsorbed $\mathrm{Zn}^{2+}$ on $\mathrm{RGO}$ with $\mathrm{S}^{2-}$ ions to generate the initial $\mathrm{ZnS}$ nuclei. An in situ charge transfer process occurs from the $\mathrm{ZnS}$ nuclei to RGO. The chemical composition and atomic weight percentage of GZ1 are shown in Fig. $6 \mathrm{~F}^{49}$ The chemical composition, atomic percentage, and percentage of weight error are given in Table 2. As the molar ratio of the $\mathrm{ZnS}$ precursor increases the chemical composition percentage increases showing the stoichiometric ratio spectroscopy recorded in a selected area of the $\mathrm{ZnS}$ architecture, indicating that it is composed of $\mathrm{Zn}$ and $\mathrm{S}$ only.

\subsection{XPS analysis}

In order to investigate the chemical composition and binding energy state of the hybrid ZnS-RGO composites, PZnS and RGO 

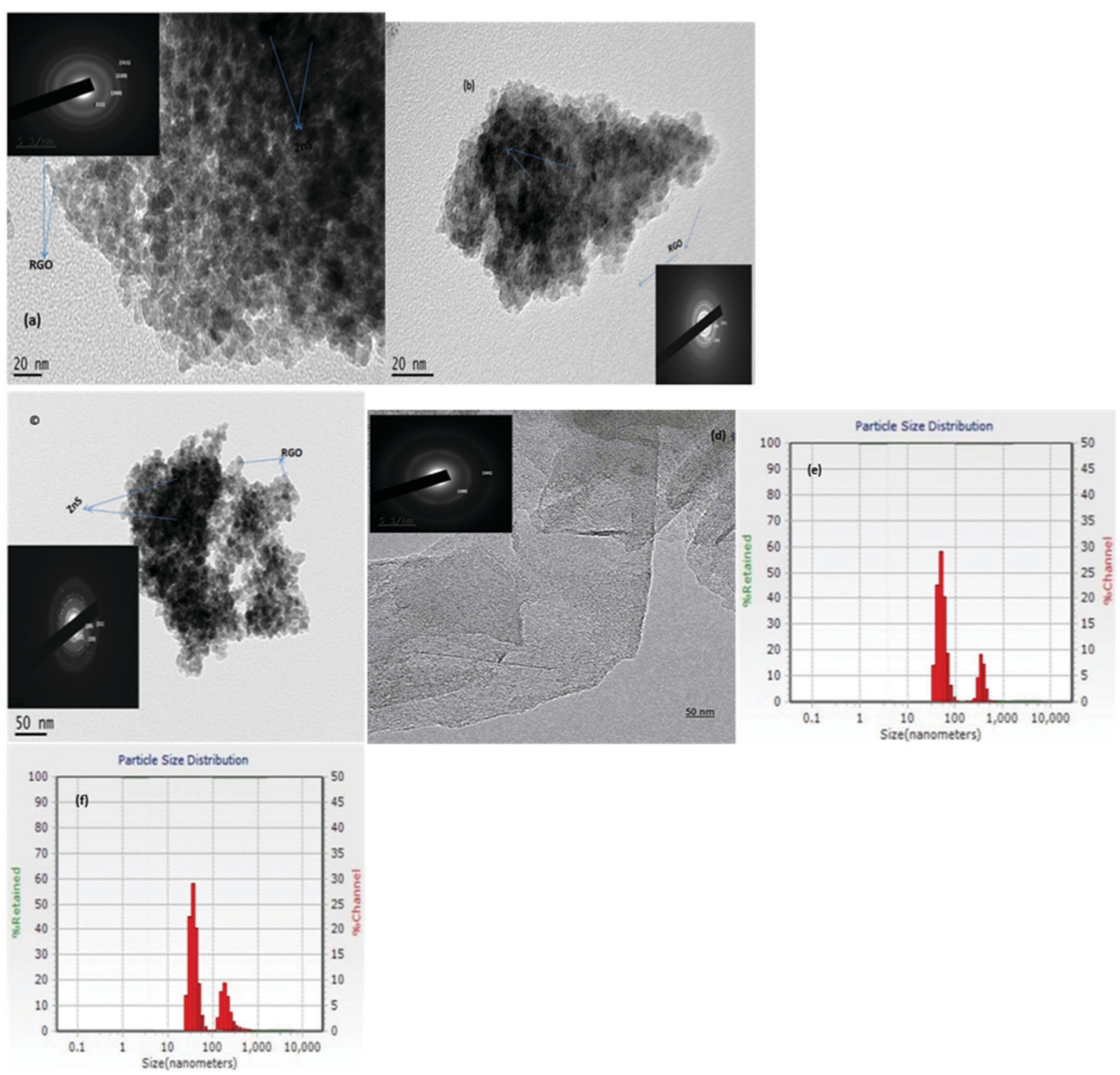

Fig. 7 TEM images of (a) GZ1, (b) GZ2, (c) GZ3 and (d) RGO, and (e and f) average distribution of particles size of the GZ1 and GZ3 composites. Inset image of (a-d) SAED of GZ1, GZ2, GZ3 and RGO.

sheets, the XPS measurement technique is employed. The overall binding energy spectra obtained from the XPS spectrum for the RGO and ZnS-RGO composite is shown in Fig. 8. Fig. 8A shows that the signal peaks of the binding energy of C1s, Zn2p, S2p and O1s are detected for ZnS-RGO (GZ1, GZ2 and GZ3) and PZnS. The deconvolution of the carbon C1s binding energy of the XPS spectra of RGO and the ZnS-RGO composites is shown in Fig. 8B. ${ }^{50}$ The binding energy peak of C1s was decomposed into peaks ranging from 282.0 to $292.0 \mathrm{eV}$, which centred at the binding energies of 289.3, 287.5, 286.3, and $284.8 \mathrm{eV}$ and were assigned to $\mathrm{C}-\mathrm{O}, \mathrm{C}-\mathrm{O}-\mathrm{C}, \mathrm{C}-\mathrm{OH}, \mathrm{C}-\mathrm{C}$ and $\Pi-\Pi^{*}(290.8 \mathrm{eV})$, respectively, which confirms the graphitic structure $\left(\mathrm{sp}^{2} \mathrm{C}-\mathrm{C}\right)$ in RGO. There is a slight right shift in the peaks of carbon bonding in the ZnS-RGO hybrid nanocomposite, which indicated the reduction of RGO and a perfect blend between ZnS and RGO in appropriate proportion as shown in Fig. 8B. This shift indicates the change in binding energies of carbon bonding with $\mathrm{Zn}$ and sulphur composition. Fig. 8(C-E) shows high resolution XPS full spectra of binding energy of Zn2p, S2p, and O1s of the ZnS-RGO (GZ1, GZ2, and GZ3) nanocomposites and PZns. ${ }^{51}$ The binding energy peaks for zinc in ZnS-RGO are located at $1021.1 \mathrm{eV}$ for the electronic state of $\mathrm{Zn} 2 \mathrm{p} 3 / 2$ and at $1044.2 \mathrm{eV}$ for the electronic state of Zn2p1/2, respectively, as shown in Fig. 8C. There is a small significant difference in the chemical shift and signal for $\mathrm{Zn} 2 \mathrm{p} 3 / 2$ and $\mathrm{Zn} 2 \mathrm{p} 1 / 2$ and intensity changes are observed indicating the chemical bonding of ZnS with RGO. The binding energy of sulphur composition is at $162 \mathrm{eV}$ and $163 \mathrm{eV}$ assigned to S2p3/2 and S2p1/2. There is a slight shift in the energy level of binding with carbon to sulphur ratio in the ZnS-RGO composites, respectively, as shown in Fig. 8D. The binding energy of oxygen composition in the composite assigned to the O1s region displayed the characteristic peaks at $532 \mathrm{eV}$ as shown in Fig. 8E. From Fig. 8E, it can observed that there is a red shift in the oxygen binding energy level compared to RGO spectra, which indicates the reduction of RGO in $\mathrm{ZnS}-\mathrm{RGO}$ composites and chemical shift of binding. ${ }^{52}$ 
Table 2 The elemental composition and atomic percentage of RGO and ZnS-RGO composites

\begin{tabular}{lllll}
\hline Sample code & Elemental line & Weight\% & Weight error\% $( \pm)$ & Atoms\% \\
\hline RGO & Carbon & 56.41 & 0.36 & 72.48 \\
& Oxygen & 33.59 & 1.40 & 27.52 \\
GZ1 & Carbon & 17.35 & 4.00 & \\
& Oxygen & 49.30 & 2.75 & 41.52 \\
& Zinc & 21.98 & 4.51 & 10.37 \\
& Sulphur & 19.10 & 1.03 & 13.00 \\
& & & & \\
GZ2 & Carbon & 10.42 & 3.91 & 23.57 \\
& Oxygen & 30.27 & 2.88 & 37.77 \\
& Zinc & 33.29 & 1.56 & 20.95 \\
& Sulphur & 21.29 & 7.93 & 14.71 \\
GZ3 & & & & \\
& Carbon & 7.42 & 3.37 & 13.15 \\
& Oxygen & 22.24 & 2.41 & 20.15 \\
& Zinc & 42.62 & 4.06 & 14.13 \\
& Sulphur & 24.72 & 1.15 &
\end{tabular}
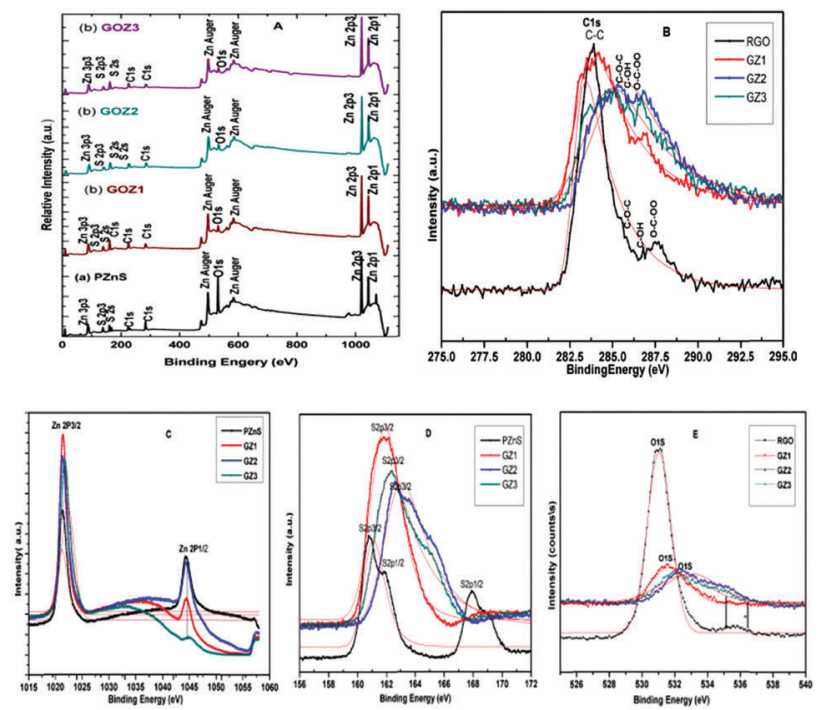

Fig. 8 XPS binding energy spectra of (A) ZnS-RGO nanocomposites (GZ1, GZ2, GZ3) and PZnS, (B) deconvolted $\mathrm{C} 1 \mathrm{~s}$ in RGO and ZnS-RGO composites (GZ1, GZ2, and GZ3), (C) XPS spectra of Zn in PZnS and ZnS-RGO composites (GZ1, GZ2, and GZ3), (D) binding energy of sulphur in PZnS and ZnS-RGO composites (GZ1, GZ2, and GZ3) and (E) binding energy of O1s in PZnS and ZnS-RGO composites (GZ1, GZ2, and GZ3).

\section{7. $\mathbf{N}_{2}$ adsorption/desorption}

Nitrogen adsorption/desorption isotherms at $77 \mathrm{~K}$ and pore size peak distribution area plots were calculated by a volumetric adsorption measurement instrument within a relative pressure range from 0.01 to $101.3 \mathrm{kPa}$. The materials were exposed to a gas mixture of $\mathrm{He}$ and $\mathrm{N}_{2}$ with a programmed ratio adsorbed amount of $\mathrm{N}_{2}$ calculated from the change of pressure in a cell after equilibrium. Before the nitrogen adsorption/desorption measurements, the samples were degassed at $300{ }^{\circ} \mathrm{C}$ under vacuum for $3 \mathrm{~h}$. Type IV adsorption isotherm of two different hysteresis loops are found in the relative pressure range of $0.0-1.0$, indicating the presence of homogeneous pores in the microporous regions. The hysteresis loop for RGO starts at 0.35 and for ZnS doped with RGO

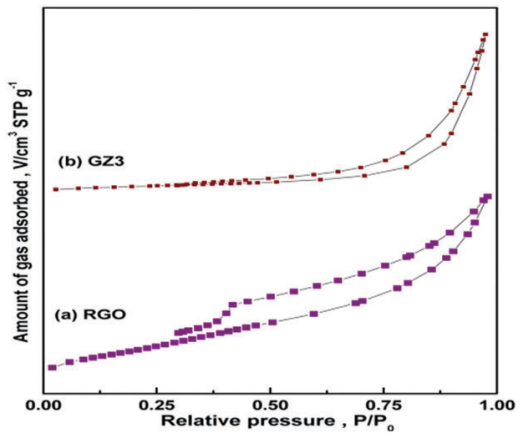

Fig. 9 Absorption/desorption graph of (a) RGO and (b) GZ3.

(GZ3) the hysteresis loop is observed at 0.275 , which indicates that the products undergo the chemical method and microwaveirradiation, reduction process technique aided with a small pore diameter of microporous nature as shown in Fig. 9. ${ }^{53,54}$

Specific surface area measurement is one of the most important characteristic tools for carbon-based materials and a promising candidate in ultracapacitors, storage, chemical/ biosensors and catalysts. The excellent microporous tendency of the synthesized samples is good for photocatalyst, water treatment, and storage applications. This confirms that the formation of hydrophobic sites and interaction between graphene sheets under irradiation conditions is the main factor in deciding the surface alteration and porous structure of RGO. The distributions of the pore size indicate that there are two types of pores in the ZnS-RGO nanocomposite. One type is concentrated in the range of a typical microporous structure of $1.25 \mathrm{~nm}$, which is probably attributed to the interspaces between the layers of RGO sheets and $\mathrm{ZnS}$ nanoparticles. The other type of pores with larger size distribution presumably arises from the spaces between nanosheets in the RGO particles. The BET surface area measurement values obtained in this study are large and indicate better porosity than the reported value of M. Sookhakian et al. $(2013)^{55}$ whose maximum value is $82.0 \mathrm{~m}^{2} \mathrm{~g}^{-1}$ and for Feng Juan Chen et al. $(2013)^{56}$ it is $160.3 \mathrm{~m}^{2} \mathrm{~g}^{-1}$. The enhanced specific surface area provides more surface active sites and efficient transport pathways to reactant molecules and products, which subsequently lead to improved photocatalytic activity of ZnS-RGO nanocomposites. Linhui Yu et al. $(2013)^{57}$ reported that the BET surface values for ZnS-graphene were $127.7 \mathrm{~m}^{2} \mathrm{~g}^{-1}$ and $149.4 \mathrm{~m}^{2} \mathrm{~g}^{-1}$ which were much less than the BET values reported in the present study. The Brunauer-Emmett-Teller specific surface areas (BET), BJH Pore volumes and average pore size of the RGO and ZnS-RGO (GZ3) are summarized in Table 3 . As it can be seen, the specific surface areas gradually increase with the increase of $\mathrm{ZnS}$ on RGO, but the average

Table 3 The surface area, mean pore diameter, total pore volume and peak radius (maximum peak distribution area of adsorption/desorption for microporous radius)

\begin{tabular}{lllll}
\hline $\begin{array}{l}\text { Sample } \\
\text { codes }\end{array}$ & $\begin{array}{l}\text { Surface } \\
\text { area }\end{array}$ & $\begin{array}{l}\text { Mean pore } \\
\text { diameter }\end{array}$ & $\begin{array}{l}\text { Total pore } \\
\text { volume }\end{array}$ & $\begin{array}{l}r_{\mathrm{p}} \\
\text { (peak radius) }\end{array}$ \\
\hline RGO & 83 & 3.15 & 0.390 & $1.72 \mathrm{~nm}$ \\
GZ3 & 182 & 2.10 & 0.196 & $1.25 \mathrm{~nm}$
\end{tabular}


pore size and pore volume decrease due to the decrease in crystallite size, which is beneficial for enhancement of the photocatalytic performance. The nanocomposites show an increase in surface area and pore size due to the enlarging area of $\mathrm{ZnS}$ and RGO. ${ }^{58}$

\subsection{Photodegradation}

To demonstrate the synergy induced enhancement of the photocatalytic efficiency of ZnS-RGO nanocomposites, contrasting experiments were performed using $\mathrm{ZnS}$ nanoparticles and RGO nanosheets as a photocatalyst for the photodegradation of BY and BB dyes respectively. The photocatalytic degradation of organic dyes by semiconductors under UV irradiation involves excitation of the semiconductors by light irradiation from photo-generated electrons in the conduction band and holes in the valence band and the subsequent chemical reactions within the surrounding media after the photo-generated charges move to the particle surface. Additionally based on the excitation of dyes in which the dyes act as a sensitizer of visible/UV light and as well as injects excited electrons to an electron acceptor to become a cationic/anion dye free radical followed by self-degradation or degradation by the reactive oxidation species. ${ }^{59}$ The results of the BY and BB degradation in a series of experimental conditions are summarized, where $C_{0}$ and $C$ are the initial concentrations after the equilibrium adsorption and the residual concentration of BY and $\mathrm{BB}$ respectively. The rate constants $k$ of photocatalytic degradation shown in Fig. 10a for 10 ppm absorption spectra are 1.05 (blank), 0.409, 0.421, 0.306, 0.375 and 0.21 for RGO, PZnS, GZ1, GZ2 and GZ3 catalysts for the final cycle of $180 \mathrm{~min}$. Similarly, for $20 \mathrm{ppm}$ the values are $0.425,0.594,0.457,0.415$ and 0.35 , respectively, for RGO, PZnS, GZ1, GZ2 and GZ3; for $30 \mathrm{ppm}$ they are $0.525,0.556,0.465,0.375$ and 0.275 , respectively, for Brilliant yellow as shown in Fig. 10(b and c) for RGO, PZnS, GZ1, GZ2 and GZ3.

The excellent photocatalytic activity of the ZnS-RGO composites is attributed to the high specific surface area and the reduction of electron-hole pair recombination due to the introduction of RGO. Thus, a large specific surface area is beneficial for absorbing more light and increasing the number of unsaturated surface coordination sites to improve the photocatalytic performance. ${ }^{66}$ In the aspect of absorption, the absorbent capacity of ZnS-RGO was obviously stronger than the bare RGO and PZnS which can be predicted from Fig. 4 and 5. As the molar ratio of ZnS precursors increases, an increase in the surface area and porosity, and narrowing/broadening of the band gap are confirmed by BET surface area measurement and UV-Vis band gap calculation in the present work. Also, the amount of RGO in ZnS-RGO increases, and the more distinct absorption capacity levelled up quickly, because of which the higher content of RGO offered a larger surface area to bind BB and BY molecules through $\Pi-\Pi$ conjugation with offset face-to-face direction. ${ }^{60}$ However, the ZnS-RGO nanocomposites were found to exhibit very prominent photocatalytic efficiency.

The rate constant $k$ values for $10 \mathrm{ppm}$ of brilliant blue are 0.7 (blank), 0.4, 0.47, 0.31, 0.25 and 0.24, respectively, for RGO, PZnS, GZ1, GZ2, and GZ3 as shown in Fig. 11a. Similarly, for $20 \mathrm{ppm}$ the values are $0.5,0.56,0.4,0.35$ and 0.3 , respectively, for RGO, PZnS, GZ1, GZ2, and GZ3. For $30 \mathrm{ppm}$ they are 0.6, 0.65, 0.47, 0.40 and 0.35 as shown in Fig. 11(b and c) for RGO, PZnS, GZ1, GZ2, and GZ3. From the results of the photodegradation experiments, it is observed that the absorption and degradation rate have lower values compared to PZNS and RGO, which indicates that the presence of RGO and $\mathrm{ZnS}$ in ZnS-RGO enriched the properties, as can be seen in Fig. 10 and 11. The degradation efficiency for the nanocomposite is better and enhanced compared to the bare RGO and $\mathrm{ZnS}$ for both the dyes (BB and $\mathrm{BY}$ ). As the molar ratio of $\mathrm{ZnS}$ increased the degradation rate also increased ${ }^{61,62}$ due to the increase in the atomic weight percentage of $\mathrm{Zn}$ to sulphur and also due to the surface plasma resonance effect and intake of energy absorption is higher in the higher molar ratio of $\mathrm{ZnS}$ on RGO. The introduction of RGO enhanced the absorption capacity of BB and BY molecules which results from the $\Pi-\Pi$ conjugation between the $\mathrm{BB}$ and $\mathrm{BY}$ and the aromatic regions of RGO.

Under UV-Vis light irradiation, electron-hole pairs are generated within $\mathrm{ZnS}$ and these photo-induced electrons are easily transferred from the ZnS conduction band to the RGO sheet via a percolation mechanism and then scavenged by dissolved oxygen which causes electron-hole separation. The improvement of ZnS-RGO composites in the photocatalysis is mainly attributed to the improvement in adsorption, which is an important prerequisite for good photocatalytic activity. ${ }^{67}$ Dye molecules can be transferred from the solution to the surface of $\mathrm{ZnS}$ and adsorbed with offset face-to-face orientation by $\Pi-\Pi$ conjugation between dyes and aromatic regions of RGO until adsorption/desorption
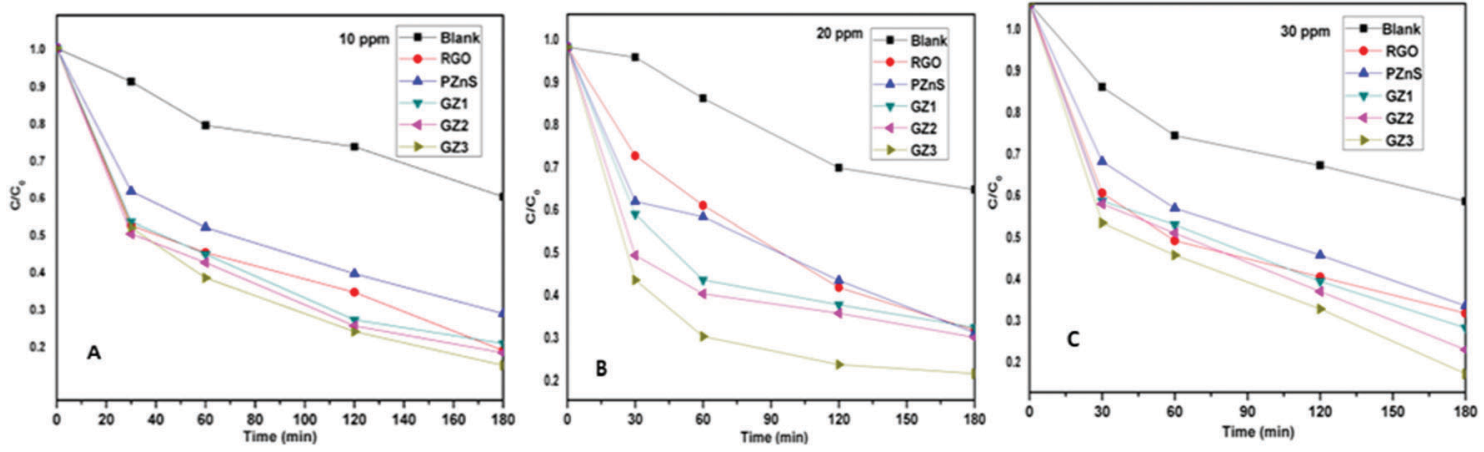

Fig. 10 The photodegradation by RGO, PZnS, GZ1, GZ2 and GZ3 of brilliant blue with different concentrations of (a) 10 ppm, (b) 20 ppm and (c) 30 ppm. 

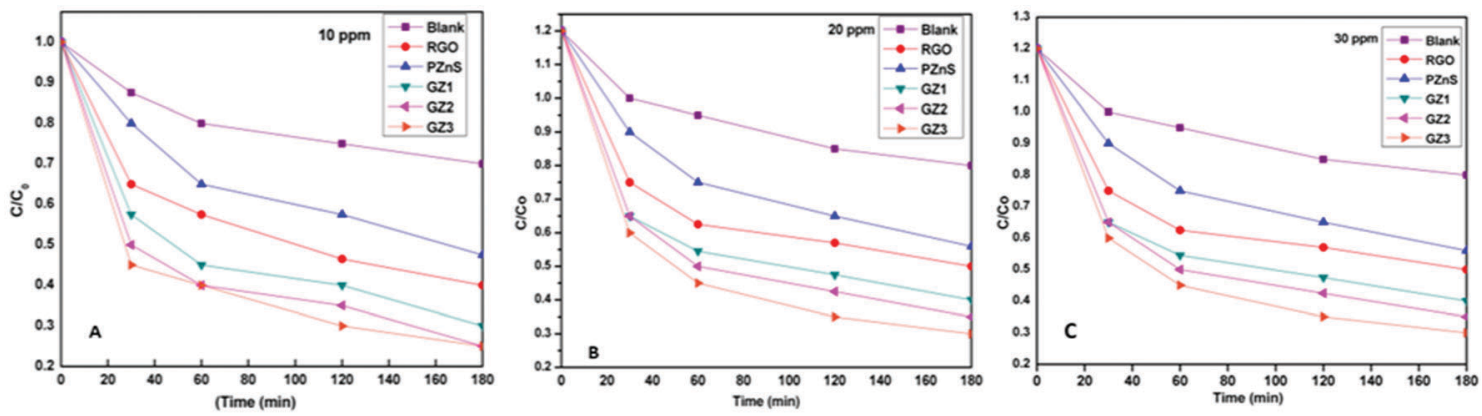

Fig. 11 The photodegradation by RGO, PZnS, GZ1, GZ2 and GZ3 of brilliant yellow with different concentrations of (a) 10 ppm, (b) 20 ppm and (c) $30 \mathrm{ppm}$.

equilibrium is achieved, ${ }^{63,66}$ the higher $\mathrm{Zn}^{2+}: \mathrm{S}^{2-}$ molar ratios and the smaller particle sizes play a vital role. A significant fluorescence quenching of $\mathrm{ZnS}$ can be observed after coupling $\mathrm{ZnS}$ with RGO due to interaction of the ZnS surface with RGO. It illustrates that the electron-hole pairs in the excited ZnS could be efficiently separated and the efficient transfer of photo-induced electrons between ZnS and RGO could take place. The highest photocatalytic activity can be attributed to the integrated effect of ZnS-RGO in which RGO in the nanocomposite not only enhances the specific surface areas of the ZnS particles but also acts as a good electron collector and transporter to separate photogenerated electron/hole pairs. Graphene has a significant influence on the morphology and structure of the nanocomposite, which can also improve the photocatalytic activity of the nanocomposite. ${ }^{64}$ The percentage of dye removal with respect to COD initial and final calculations is given in Table 4 .

\subsection{Photodegradation mechanism}

The enhanced photodegradation efficiency of the ZnS-RGO composites is attributed to the adsorption of contaminant molecules by catalysis, the light absorption and finally the charge separation and transportation. The optical quenching and surface resonance characteristics of $\mathrm{ZnS}$ are higher compared to the ZnS-RGO composite and efficiency enhancement of the ZnS-RGO composite is due to the extended light absorption range. A significant fluorescence quenching of $\mathrm{ZnS}$ can be observed after coupling ZnS with RGO due to interaction of the $\mathrm{ZnS}$ surface with RGO compared to the bare ZnS and a clear red shift in the absorption edge of the ZnS-RGO composite can be predicted from Fig. 4 and 5, which gives confirmation of the photo-responsive nature of the materials. ${ }^{66}$ The adsorption

Table 4 Percentage removal of dye concentration for BB and BY

\begin{tabular}{llllllll}
\hline & \multicolumn{3}{l}{$\begin{array}{l}\text { \% Removal dye } \\
\text { concentration (BB) }\end{array}$} & & \multicolumn{3}{l}{$\begin{array}{l}\text { \% Removal dye } \\
\text { concentration (BY) }\end{array}$} \\
\cline { 2 - 4 } Samples & $10 \mathrm{ppm}$ & $20 \mathrm{ppm}$ & $30 \mathrm{ppm}$ & & $10 \mathrm{ppm}$ & $20 \mathrm{ppm}$ & $30 \mathrm{ppm}$ \\
\hline RGO & 66.59 & 60.15 & 55.89 & & 70.25 & 66.03 & 63.59 \\
PZnS & 55.7 & 50.52 & 50.68 & & 60.27 & 57.52 & 55.7 \\
GZ1 & 70.02 & 65.86 & 63.60 & & 75.64 & 73.63 & 70.02 \\
GZ2 & 78.65 & 77.31 & 72.35 & & 80.68 & 76.31 & 74.65 \\
GZ3 & 82.89 & 80.25 & 80.76 & & 87.61 & 82.99 & 80.89
\end{tabular}

activity of the dyes is enhanced for the ZnS-RGO composite compared to PZnS as can be seen in Fig. 10 and 11.

$\mathrm{ZnS}$ valence electrons are excited to the conduction band, thereby producing the electron-hole pairs. The photo-generated electrons are transferred to RGO, which acts as an electron acceptor. RGO promotes the interfacial electron transfer process and effectively hinders the recombination of the photo-generated electrons and holes. The holes in the valence band of $\mathrm{ZnS}$ can react with absorbed water or hydroxyl groups to form surface hydroxyl radicals. Meanwhile, the photo-generated electrons on the surface of the $\mathrm{ZnS}$ nanoparticles, as well as the trapped electrons on RGO, can also react with the dissolved oxygen to form reactive oxygen species, which further react with water to form hydroxyl radicals. ${ }^{64}$ Both the hydroxyl radicals and holes can degrade and oxidize the dye (BB and BY) molecules. ${ }^{62,65,66}$ Electron-hole pairs in the excited ZnS could be efficiently separated and the efficient transfer of photoinduced electrons between ZnS and RGO plays a vital role in degradation. The efficiency of the photocatalytic reaction significantly depends on the efficiency of adsorption of organic contaminants on the photocatalysts and the separation of the photo-generated electron-hole pairs. The holes can react either with adsorbed water or with surface hydroxyl to form hydroxyl radicals. Consequently, the adsorption equilibrium is destroyed and dye molecules could move from solution to the interface and consequently decompose to $\mathrm{CO}_{2}, \mathrm{H}_{2} \mathrm{O}$ and other minerals through a redox reaction. The mechanism of photodegradation of the dyes is illustrated in Fig. 12.

$$
\mathrm{RGO}+\mathrm{e}^{-} \rightarrow \mathrm{RGO}\left(\mathrm{e}^{-}\right), \mathrm{ZnS}+h \nu \rightarrow \mathrm{ZnS}\left(\mathrm{h}^{+}+\mathrm{e}^{-}\right)
$$

$\mathrm{RGO}\left(\mathrm{e}^{-}\right)+\mathrm{O}_{2} \rightarrow \mathrm{RGO}+{ }^{\bullet} \mathrm{O}_{2}^{-}, \mathrm{RGO}\left(\mathrm{e}^{-}\right)+\mathrm{ZnS}(\mathrm{h}) \rightarrow$ photons

$$
\mathrm{h}^{+}+\mathrm{OH}^{-} \rightarrow \cdot \mathrm{OH}
$$

$$
\cdot \mathrm{OH}+\text { dyes } \rightarrow \text { degradation }\left(\mathrm{CO}_{2}, \mathrm{H}_{2} \mathrm{O}\right)
$$

Under UV light, ZnS emitted to produce photoelectrons and holes. ${ }^{64}$ The photoelectrons used for degrading dyes must be accepted and delivered away to resist the recombination of the photoelectrons and holes, which would give a higher efficiency of photocatalytic degradation. Fortunately, RGO can offer access 


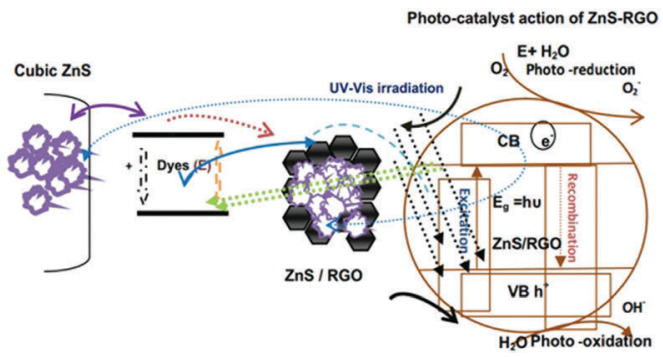

Fig. 12 Mechanism of photodegradation of dyes.

to accept and transfer the photoelectrons, which was proven by the PL as shown in Fig. 5. Another key factor for the high performance of photocatalysts is that the $\mathrm{ZnS}$ nanostructure and RGO sheets can connect to each other with very tight bonding. Such a photocatalytic degradation process for dyes has been demonstrated to be an efficient approach to remove textile dye stuff from aquatic environments.

\section{Experimental procedure}

\subsection{Materials}

Graphite powder ( -300 mesh, 99.95\%, MERCK), 95\% hydrochloric acid $(\mathrm{HCl}), 98 \%$ sulphuric acid $\left(\mathrm{H}_{2} \mathrm{SO}_{4}\right), 95 \%$ phosphoric acid $\left(\mathrm{H}_{3} \mathrm{PO}_{4}\right), 30 \%$ hydrogen peroxide $\left(\mathrm{H}_{2} \mathrm{O}_{2}\right), 95 \%$ potassium permanganate $\left(\mathrm{KMnO}_{4}\right)$, zinc sulphate $\left(\mathrm{ZnSO}_{4} \cdot 5 \mathrm{H}_{2} \mathrm{O}\right)$, sodium sulphide $\left(\mathrm{Na}_{2} \mathrm{~S} \cdot 7 \mathrm{H}_{2} \mathrm{O}\right)$ and ethanol were purchased from SigmaAldrich Chemical Reagent Co. Ltd.

\subsection{Preparation of $\mathrm{ZnS}$}

For the synthesis of $\mathrm{ZnS}=\left(1 \mathrm{~mol}, 3.6 \mathrm{~g}, \mathrm{ZnSO}_{4} \cdot 5 \mathrm{H}_{2} \mathrm{O}\right)$ and ( $1 \mathrm{~mol}, 1.56 \mathrm{~g}, \mathrm{Na}_{2} \mathrm{~S} \cdot 7 \mathrm{H}_{2} \mathrm{O}$ ) powders used as precursor materials were dissolved separately in $20 \mathrm{~mL}$ of deionized water and stirred at room temperature for $30 \mathrm{~min}$. Then, the solutions were mixed by dripping the $\mathrm{Na}_{2} \mathrm{~S}$ solution in drops into $\mathrm{ZnSO}_{4}$ solution while stirring at $80{ }^{\circ} \mathrm{C}$ for 2 hours. A white gel formed and was treated with microwave radiation for $180 \mathrm{~s}$ at $450 \mathrm{~W}$. The products obtained were washed several times with deionized water and ethanol followed by centrifuging at $4000 \mathrm{rpm}$ for $20 \mathrm{~min}$. The final products were dried in a vacuum oven for $24 \mathrm{~h}$ at $80{ }^{\circ} \mathrm{C}$ to yield (pure) PZnS.

\subsection{Synthesis of reduced graphene oxide}

Reduced graphene oxide (RGO) is prepared from natural graphite powder by an improved Hummer's method ${ }^{27}$ and a chemical exfoliation process. In brief, a 9:1 pre-cooled mixture of conc. $\mathrm{H}_{2} \mathrm{SO}_{4} / \mathrm{H}_{3} \mathrm{PO}_{4}$ is slowly poured under continuous stirring into a mixture of $1 \mathrm{~g}$ of natural graphite powder and $0.5 \mathrm{~g}$ of $\mathrm{NaNO}_{3}$ powder in a $1 \mathrm{~L}$ conical flask. Then $6 \mathrm{~g}$ of $\mathrm{KMnO}_{4}$ is slowly added in steps maintaining the temperature of the mixture at $25{ }^{\circ} \mathrm{C}$, and stirring continuously for 4 hours produces an exothermic reaction by which a rise in temperature up to $80{ }^{\circ} \mathrm{C}$ to $100{ }^{\circ} \mathrm{C}$ is observed. Allow the solution to cool down to room temperature, now gradually add $280 \mathrm{~mL}$ of DI water containing $5 \mathrm{~mL}$ of $30 \% \mathrm{H}_{2} \mathrm{O}_{2}$ and keep it overnight for decantation to form a solid precipitation. The products were washed 5 to 7 times with $5 \%$ warm solution of $\mathrm{HCl}$ to remove the metal ions and impurities. This mixture was centrifuged for $30 \mathrm{~min}$ at $4000 \mathrm{rpm}$ followed by ethanol wash and filtered to obtain a black powder. Finally, the black powder is vacuum dried at a temperature of $100{ }^{\circ} \mathrm{C}$ for 24 hours to obtain pure reduced graphene oxide.

\subsection{Synthesis of ZnS-RGO nanocomposites}

To synthesise ZnS-RGO nanocomposites, $0.2 \mathrm{~g}$ of RGO was dispersed in $100 \mathrm{~mL}$ of deionized water and ultrasonication was done for $60 \mathrm{~min}$ to form a homogenous solution and for further exfoliation of reduced graphene oxide. Then, $1 \mathrm{M}$ of ZnS-RGO nanocomposite $(\mathrm{GZ3})=$ zinc sulphate $\left(1 \mathrm{~mol}, 3.62 \mathrm{~g}, \mathrm{ZnSO}_{4}\right)$ and sodium sulphide ( $1 \mathrm{~mol}, 1.56 \mathrm{~g}, \mathrm{Na}_{2} \mathrm{~S}$ ) were gradually dissolved in the above prepared RGO solution at $80{ }^{\circ} \mathrm{C}$ under stirring conditions for $1 \mathrm{~h}$. Similarly, $0.5 \mathrm{M}$ and $0.25 \mathrm{M}$ of $\mathrm{ZnS}$ nanoparticles were dispersed in $0.2 \mathrm{~g}$ of the above sonicated RGO solution to form hybrid nanocomposites namely GZ1 and GZ2. The nanocomposite solution was exposed to microwave radiation for $5 \mathrm{~min}$ at $450 \mathrm{~W}$ and the final products (irradiated solution from the microwave vessel is slowly poured into a glass beaker after washing followed by filtering) were kept for drying at $80{ }^{\circ} \mathrm{C}$ in a vacuum oven to obtain hybrid ZnS-RGO nanocomposites.

\subsection{Characterization}

The phase and crystallite size of the ZnS nanoparticles, RGO sheets, and ZnO-RGO nanocomposites were characterized using an automated X-ray powder diffractometer (XRD, Rigaku smart Lab-II) with mono-chromatic $\operatorname{CuK} \alpha(\lambda=1.544 \AA)$ radiation. The structural and morphology characterization of the synthesized products were analyzed using a field emission scanning electron microscope (FE-SEM, Carl Zeiss, sigma 500) and a transmission electron microscope (TEM, JEOL JEM-2100F-HR) equipped with a field emission gun operated at $200 \mathrm{kV}$. The images were recorded by using a Gatan Orius SC 1000 (832) model. The chemical compositions and binding energy of the nanocomposites were determined by using a Kratos Axis, ULTRA X-ray Photoelectron Spectrometer incorporating a $165 \mathrm{~mm}$ hemispherical electron energy analyser. Energy dispersive X-ray spectroscopy analysis (EDS) using an EDS-System (Hitachi-S3400N) instrument attached to the SEM was employed to investigate the elemental composition of the samples. The optical diffuse reflectance absorption spectra were recorded using a UV-Vis spectrophotometer (Diode Array spectrometer, SA-165, Elico, India). The Fourier Transform Infrared (FTIR) analyses were performed by using an FTIR460 plus spectrophotometer (Jasco, Japan) at wave numbers ranging from 4000 to $400 \mathrm{~cm}^{-1}$. The Micro-Raman spectroscopy was performed using an SP-acton 2500 and an argon laser lamp of $\lambda=465 \mathrm{~nm}$ excitation. Photoluminescence spectra (PL) were recorded by using an F-2700 FL spectrophotometer with an incident laser light of $375 \mathrm{~nm}$ wavelength. The average particle size distribution was calculated by the dynamic light scattering technique (DLS, Nanotrac wave, Microtrac, Japan). 


\subsection{Photocatalytic experiment}

The photocatalytic activities of the synthesized materials were evaluated by the degradation of Brilliant Yellow (BY) and Brilliant Blue (BB) dyes $(200 \mathrm{~mL}$ of aqueous solution of the dyes $\left.\left(1 \times 10^{-5} \mathrm{~mol} \mathrm{~L}^{-1}\right)\right)$ under UV light radiation. The light source used was a $150 \mathrm{~W}$ Xe (Xenon) lamp and the distance between the UV source and the photoreaction vessel was $10 \mathrm{~cm}$. Prior to irradiation, the suspensions were magnetically stirred in the dark for $30 \mathrm{~min}$ and further the photoreaction vessel was exposed to UV irradiation under ambient conditions. Different concentrations of dyes (10 ppm, $20 \mathrm{ppm}$, and $30 \mathrm{ppm}$ ) were considered for performing the photodegradation experiment with $10 \mathrm{mg}$ of catalyst nanoparticles. At regular time intervals, $3 \mathrm{~mL}$ of the suspension was taken for centrifugation to separate the photocatalyst and further evaluated using a UV-Vis absorption spectrometer.

The photodegradation efficiency percentage is calculated from the equation given below

$$
\% \text { Photodegradation efficiency }=\frac{C_{0}-C}{C_{0}} \times 100
$$

where $C_{0}=$ initial concentration of dye and $C=$ concentration of dye after photoirradiation (final).

\section{Conclusion}

ZnS-RGO nanocomposites have been successfully synthesized using a sol-gel method without using any surfactant/stabilizing agent and were treated with the microwave irradiation technique. The XRD patterns of the ZnS-RGO nanocomposite shows that the crystallinity and particle size well support the TEM images. The FESEM and TEM images of the ZnS-RGO composites show that ZnS nanoparticles are in a cubic/isometric lattice structure decorated on graphene sheets with high crystallinity and fine morphology. FTIR and Raman results evidently show the presence of $\mathrm{ZnS}$, RGO and the reduction of reduced graphene oxide in the composite to graphene. The ZnS-RGO composite gives significant improvement in the degradation of BY and BB dyes under UV-Vis irradiation, compared to PZnS and RGO which is due to increased adsorption of the dye, an increase in the band gap and the stepwise structure of the energy levels in the composite. The highest photocatalytic activity can be attributed to the integrated effect in which RGO in the nanocomposite not only enhances the specific surface areas of the ZnS particles, but also acts as a good electron collector and transporter to separate photo-generated electron/hole pairs. RGO has a significant influence on the morphology and structure of the nanocomposite, which can also improve the photocatalytic activity of the nanocomposite. Overall in this research work, in situ fabrication of ZnS-RGO composites with good efficiency photocatalysis under UV-Visible light irradiation and their applications in environmental protection are achieved.

\section{Acknowledgements}

Department of Science and Technology, India funded this research work. The authors acknowledge Prof. M. K. Kokila, Bangalore University for help in providing photoluminescence spectroscopy studies. We are thankful to the University of South Australia for providing the XPS and TEM characterization.

\section{Notes and references}

1 C. Y. Park, J. G. Choi, T. Ghosh, Z.-D. Meng, L. Zhu and W. C. Oh, Fullerenes, Nanotubes, Carbon Nanostruct., 2014, 22, 630-642.

2 M. R. Hoffmann, S. T. Martin, W. Choi and D. W. Bahnemann, Chem. Rev., 1995, 95, 69-96.

3 Q. Xiang, J. Yu, B. Cheng and H. Ong, Chem. - Asian J., 2010, 5, 1466-1474.

4 J. Yu, Q. Xiang, J. Ran and S. Mann, CrystEngComm, 2010, 12, 872-879.

5 H. Zhang, R. Xie, T. Sekiguchi, X. Ma and D. Yang, Mater. Res. Bull., 2007, 42, 1286-1292.

6 A. B. Panda, G. Glaspell and M. S. El-Shall, J. Am. Chem. Soc., 2006, 128, 2790-2791.

7 J. F. Shen, M. Shi, N. Li, B. Yan, H. W. Ma, Y. Z. Hu and M. X. Ye, Nano Res., 2010, 3, 339-349.

8 H. Hu, X. Wang, F. Liu, J. Wang and C. Xu, Synth. Met., 2011, 161, 404-410.

9 X. Fang, T. Zhai, U. K. Gautam, L. Li, L. Wu, Y. Bando and D. Golberg, Prog. Mater. Sci., 2011, 56, 175-287.

10 G. Li, J. Zhai, D. Li, X. Fang, H. Jiang and Q. Dong, J. Mater. Chem., 2010, 20, 9215-9219.

11 S. H. Yu and M. Yoshimura, Adv. Mater., 2002, 14, 296-300.

12 S. Kar, S. Biswas and S. Chaudhuri, Nanotechnology, 2005, 16, 737-740.

13 D. G. Chen, F. Huang, G. Q. Ren, D. S. Li, M. Zheng, Y. J. Wang and Z. Lin, Nanoscale, 2010, 2, 2062-2066.

14 J. Shang, M. Chai and Y. Zhu, J. Solid State Chem., 2003, 174, 104-110.

15 R. Czerw, B. Foley, D. Tekleab, A. Rubio, P. M. Ajayan and D. L. Carroll, Phys. Rev. B: Condens. Matter Mater. Phys, 2002, 66, 334081.

16 S. J. Park and R. S. Ruoff, Nat. Nanotechnol., 2009, 4, 217-224.

17 A. K. Geim, Science, 2009, 324, 1530-1534.

18 K. Geim and K. S. Novoselov, Nat. Mater., 2007, 6, 183.

19 J. Yu, H. Yu, B. Cheng, X. Zhao and Q. Zhang, J. Photochem. Photobiol., A, 2006, 182, 121-127.

20 P. Wang, T. F. Jiang, C. Z. Zhu, Y. M. Zhai, D. J. Wang and S. J. Dong, Nano Res., 2010, 3, 794-799.

21 G. Wang, Q. Tang, H. Bao, X. Li and G. Wang, J. Power Sources, 2013, 241, 231-238.

22 A. Ramadoss and S. J. Kim, Carbon, 2013, 63, 434-445.

23 M. Feng, R. Sun, H. Zhan and Y. Chen, Nanotechnology, 2010, 21, 075601.

24 S. D. Miao, Z. M. Liu, B. X. Han, H. W. Yang, Z. J. Miao and Z. Y. Sun, J. Colloid Interface Sci., 2006, 301, 116-122. 
25 K. Lellala, K. Namratha and K. Byrappa, Mater. Today: Proc., 2016, 3, 74-83.

26 U. Alver, W. Zhou, A. B. Belay, R. Krueger, K. O. Davis and N. S. Hickman, Appl. Surf. Sci., 2012, 258, 3109-3114.

27 W. S. Hummers and R. E. Offeman, J. Am. Chem. Soc., 1958, 98, 1339.

28 M. Muruganandham, R. Amutha and M. Sillanpaa, ACS Appl. Mater. Interfaces, 2010, 2, 1817-1823.

29 C. Nethravathi, T. Nisha, N. Ravishankar, C. Shivakumara and M. Rajamathi, Carbon, 2009, 47, 2054-2059.

30 X. J. Lv, W. F. Chang, H. X. Fu, H. Zhang, J. S. Cheng, G. L. Zhang, Y. Song, C. Y. Hu and J. H. Li, J. Mater. Chem., 2012, 22, 1539-1546.

31 H. K. Jeong, Y. P. Lee, R. J. Lahaye, M. H. Park, K. H. An, I. J. Kim, C. W. Yang, C. Y. Park, R. S. Ruoff and Y. H. Lee, J. Am. Chem. Soc., 2008, 130, 1362-1366.

32 S. Stankovich, D. A. Dikin, R. D. Piner, K. A. Kohlhaas, A. Kleinhammes, Y. Jia, Y. Wu, S. T. Nguyen and R. S. Ruoff, Carbon, 2007, 45, 1558-1565.

33 S. Pan and X. Liu, J. Solid State Chem., 2012, 191, 51-56.

34 H. Hu, X. Wang X, F. Liu, J. Wang J and C. Xu, Synth. Met., 2011, 161, 404-410.

35 L. J. Yao, M. J. Zheng, S. H. He, L. Ma, M. Li and W. Z. Shen, Appl. Surf. Sci., 2011, 257, 2955-2959.

36 K. N. Kudin, B. Ozbas, H. C. Schniepp, R. K. Prudhomme, I. A. Aksay and R. Car, Nano Lett., 2008, 8, 36-41.

37 D. Denzler, M. Olschewski and K. Sattler, J. Appl. Phys., 1998, 84, 2841-2845.

38 X. Wang, L. Zhi and K. Müllen, Nano Lett., 2008, 8, 323-327.

39 H. Hu, X. Wang, F. Liu, J. Wang and J. C. Xu, Synth. Met., 2011, 161, 404-410.

40 B. Meyer, Chem. Rev., 1976, 76, 367-388.

41 Y. Mikhlin, M. Likhatski, A. Karacharov, V. Zaikovski and A. Krylov, Phys. Chem. Chem. Phys., 2009, 11, 5445-5454.

42 P. Wang, T. Jiang, C. Zhu, Y. Zhai, D. Wang and S. Dong, Nanoscale, 2010, 3, 794-799.

43 N. Chestnoy, T. D. Harris, R. Hull and L. E. Brus, J. Chem. Phys., 1986, 90, 3393-3399.

44 V. Gupta, N. Chaudhary, R. Srivastava, G. D. Sharma, R. Bhardwaj and S. Chand, J. Am. Chem. Soc., 2011, 133, 9960-9963.

45 L. Zhu, Z. D. Meng, M. L. Chen, F. L. Zhang, J. G. Choi, J. Y. Park and W. C. Oh, J. Photocatal. Sci., 2010, 1, 69-76.

46 Y. J. Wang, R. Shi, J. Lin and Y. F. Zhu, Appl. Catal., B, 2010, 100, 353-359.
47 Q. Xiang, J. Yu and M. Jaroniec, Nanoscale, 2011, 3, 3670-3678.

48 C. Xu, X. Wang and J. W. Zhu, J. Phys. Chem. C, 2008, 112, 19841-19845.

49 D. Y. Cai and M. Song, J. Mater. Chem., 2007, 17, 3678-3680.

50 K. Lellala, K. Namratha and K. Byrappa, Microporous Mesoporous Mater., 2016, 226, 522-529.

51 Y. H. Ng, A. Iwase, A. Kudo and R. Amal, J. Phys. Chem. Lett., 2010, 1, 2607-2612.

52 A. Peigney, C. H. Laurent, E. Flahaut, R. Bacsa and A. Rousset, Carbon, 2001, 39, 507-514.

53 A. B. Bourlinos, T. A. Steriotis, M. Karakassides, Y. Sanakis, V. Tzitzios and C. Trapalis, et al., Carbon, 2007, 45, 852-857.

54 Y. Gao, D. Ma, C. Wang, J. Guan and X. Bao, Chem. Commun., 2011, 47, 2432-2434.

55 M. Sookhakian, Y. M. Amin and W. J. Basirun, Appl. Surf. Sci., 2013, 283, 668-677.

56 F. J. Chen, Y. L. Cao and D. Z. Jia, CrystEngComm, 2013, 15, 4747-4754.

57 L. Yu, H. Ruan, Y. Zheng and L. Danzhen, Nanotechnology, 2013, 24, 37560.

58 Y. Xu, K. Sheng, C. Li and G. Shi Self, ACS Nano, 2010, 4, 4324-4330.

59 L. Zhu, Z. D. Meng, M. L. Chen, F. J. Zhang, J. G. Choi, J. Y. Park and W. C. Oh, J. Photocatal. Sci., 2010, 1, 69-76.

60 S. Stankovich, D. A. Dikin, G. H. B. Dommett, K. M. Kohlhass, E. J. Zimney, E. A. Stach, R. D. Piner, S. T. Nguyen and R. S. Ruoff, Nature, 2006, 442, 282-286.

61 Z. Y. Gao, N. Liu, D. P. Wu, W. G. Tao, F. Xu and K. Jiang, Appl. Surf. Sci., 2012, 258, 2473-2478.

62 D. Xu, B. Cheng, S. Cao and J. Yu, Appl. Catal., B, 2015, 164, 380-388.

63 Y. Wang, J. Yu, W. Xiao and Q. Li, J. Mater. Chem. A, 2014, 2, 3847-3855.

64 Q. Xiang, J. Yu and M. Jaroniec, Chem. Soc. Rev., 2012, 41, 782-796.

65 L. Kashinath, K. Namratha and K. Byrappa, Appl. Surf. Sci., 2015, 357, 1849-1856.

66 Y. Zhang, N. Zhang, Z.-R. Tang and Y.-J. Xu, ACS Nano, 2012, 6, 9777-9789.

67 Y. H. Zhang, Z. R. Tang, X. Z. Fu and Y. J. Xu, ACS Nano, 2010, 4, 7303-7314.

68 W. Tu and H. Liu, J. Mater. Chem., 2000, 10, 2207-2211.

69 P. Lidstrom, J. Tierney, B. Wathey and J. Westman, Tetrahedron, 2001, 57, 9225-9283. 